\title{
Maximum A Posteriori Estimation of Isotropic High-Resolution Volumetric MRI from Orthogonal Thick-Slice Scans
}

\author{
Ali Gholipour, Judy A. Estroff, Mustafa Sahin, \\ Sanjay P. Prabhu, and Simon K. Warfield \\ Departments of Radiology and Neurology, Childrens Hospital Boston, \\ and Harvard Medical School, 300 Longwood Ave., Boston, MA 02115, \\ http://www.crl.med.harvard.edu/
}

\begin{abstract}
Thick-slice image acquisitions are sometimes inevitable in magnetic resonance imaging due to limitations posed by pulse sequence timing and signal-to-noise-ratio. The estimation of an isotropic highresolution volume from thick-slice MRI scans is desired for improved image analysis and evaluation. In this article we formulate a maximum a posteriori (MAP) estimation algorithm for high-resolution volumetric MRI reconstruction. As compared to the previous techniques, this probabilistic formulation relies on a slice acquisition model and allows the incorporation of image priors. We focus on image priors based on image gradients and compare the developed MAP estimation approach to scattered data interpolation (SDI) and maximum likelihood reconstruction. The results indicate that the developed MAP estimation approach outperforms the SDI techniques and appropriate image priors may improve the volume estimation when the acquired thick-slice scans do not sufficiently sample the imaged volume. We also report applications in pediatric and fetal imaging.
\end{abstract}

Keywords: MAP estimation, super-resolution, volume reconstruction.

\section{Introduction}

Thick slice image acquisitions are sometimes inevitable in magnetic resonance imaging (MRI) due to pulse sequence timing requirements and the need to maintain high signal-to-noise-ratio (SNR). Such scans are typically performed for T2weighted and diffusion weighted imaging in a variety of applications including brain, lung, and heart imaging, and fetal and neonatal MRI. Thick-slice scans are acquired in single shot fast spin echo (SSFSE) imaging of fetuses, neonates, and pediatric patients who may move in the scanner [1]. SSFSE slices are acquired in a fraction of a second, thus freezing the motion of the subject. Nevertheless, due to thick slice acquisitions necessary to maintain SNR and the inter-slice motion artifacts, these images do not appropriately reflect the 3D anatomy.

The reconstruction of a high-resolution volumetric image from thick slice scans is desired for enhanced image analysis and improved evaluation. Inter-slice reconstruction has been previously addressed in [2] based on an iterative back 
projection reconstruction algorithm, where multiple shifted thick-slice scans provide dense sampling of the imaged object. In more recent studies [1], 3], 4] high-resolution volumes have been reconstructed from fast slice scans of moving subjects. These scans are affected by inter-slice motion thus iterations of sliceto-volume registration and scattered data interpolation (SDI) have been used in these studies for iterative motion estimation and volume reconstruction.

Nevertheless, scattered data interpolation techniques do not provide a mathematical framework to justify that the estimated high-resolution volume is a minimum error representation of the imaged object given the acquired scans. In this article we have formulated a general mathematical framework based on a maximum a posteriori (MAP) estimation algorithm for high-resolution volume reconstruction. Inspired by the recent advances in super-resolution image reconstruction [5, 6], the developed MAP estimation approach relies on a slice acquisition model and minimizes a cost function of the error norm between the estimated volume and the acquired slices.

In addition, the MAP estimation approach allows the incorporation of prior image models for volume reconstruction, which is critical when the number of thick-slice scans is limited and the slice thickness is significantly larger than the matrix resolution. Under certain conditions the developed MAP estimation approach simplifies to regularized MLE reconstruction, thus the main contribution in this article is the development and performance analysis of MAP volume estimation and comparison to SDI and non-regularized MLE solutions.

As such, we focus on simple image priors based on image gradients for performance analysis of high-resolution volume reconstruction using the formulated MAP estimation approach. We limit our evaluation to the reconstruction of isotropic brain volumes from a limited number of orthogonal thick-slice scans, but the results can be generalized to similar applications. Our evaluation involves quantitative analysis using synthetic digital brain phantom images, and applications in pediatric and fetal MRI. We compare the MAP estimation technique with the B-Spline SDI approach in [1] and a non-regularized MLE solution, and evaluate the effect of image priors under different scanning conditions.

\section{Methods}

In order to formulate the volume estimation problem in a super-resolution framework we need to establish a slice acquisition model, which describes how the acquired slices are obtained from the imaged object. The following slice acquisition model is considered in this study:

$$
\mathbf{y}_{k}=\mathbf{D}_{k} \mathbf{B}_{k} \mathbf{S}_{k} \mathbf{M}_{k} \mathbf{x}+\mathbf{v}_{k} ; k=1, \ldots, n
$$

where $\mathbf{y}_{k}$ is the vector of the voxels of the $k^{\text {th }} 2 \mathrm{D}$ slice with slice thickness $\Delta s_{k}$ and uniform in-plane spacing of $\Delta \rho_{k} ; \mathbf{x}$ is a vector of the desired reconstructed image voxels in the lexicographical order with isotropic spacing of $\Delta \rho ; \mathbf{v}_{k}$ is the residual noise vector, $n$ is the number of slices obtained from $N$ scans, $\mathbf{M}_{k}$ is the matrix of motion parameters, $\mathbf{S}_{k}$ is a matrix representing the slice selection 
profile, $\mathbf{B}_{k}$ is a blur matrix representing the point spread function (PSF) of the MRI signal acquisition process, and $\mathbf{D}_{k}$ is a down-sampling matrix.

On the basis of Equation (11) the imaged object goes through geometric and signal operations, including motion, slice selection and signal averaging, PSF blur, and resampling, to generate the acquired slices. Assuming that all the matrix operations, including the motion parameter matrices are known in Equation (1), this equation can be written in a simple linear form like $\mathbf{y}_{k}=\mathbf{W}_{k} \mathbf{x}+\mathbf{v}_{k}$, where $\mathbf{W}_{k}=\mathbf{D}_{k} \mathbf{B}_{k} \mathbf{S}_{k} \mathbf{M}_{k}$. These linear equations can be augmented to form a large linear matrix equation like $\mathbf{y}=\mathbf{W} \mathbf{x}+\mathbf{v}$. Super-resolution volume reconstruction is the inverse problem of finding $\mathbf{x}$ given the acquired slices $\mathbf{y}_{k}$. The classical solution to this linear inverse problem can be obtained through maximum likelihood estimation (MLE).

The MAP estimation is considered as a generalization of MLE and is written based on the conditional probability density function (PDF) of the acquired slices $\mathbf{y}_{k}$ given the estimated volume $\hat{\mathbf{x}}$ as well as the prior information about the $\mathrm{PDF}$ of the estimated volume, i.e. $\operatorname{Pr}(\hat{\mathbf{x}})$ :

$$
\mathbf{x}_{\mathrm{MAP}}=\arg \max _{\mathbf{x}}\left[\log \operatorname{Pr}\left(\mathbf{y}_{k} \mid \hat{\mathbf{x}}\right)+\log \operatorname{Pr}(\hat{\mathbf{x}})\right]
$$

The MAP solution depends on the probability functions. Here we assume that the noise residuals (error samples) are drawn from Gaussian distributions with mean of zero and standard deviation of $\sigma_{k}$. Therefore:

$$
\operatorname{Pr}\left(\mathbf{y}_{k} \mid \hat{\mathbf{x}}\right)=\prod_{i} \frac{1}{\sigma_{k} \sqrt{2 \pi}} \exp \left(-\frac{\left(\hat{y}_{k}(i)-y_{k}(i)\right)^{2}}{2 \sigma_{k}^{2}}\right)
$$

where $y_{k}(i)$ are the samples from the acquired slices $\mathbf{y}_{k}$, and $\hat{y}_{k}(i)$ are the samples from the estimated slices $\hat{\mathbf{y}}_{k}=\mathbf{W}_{k} \hat{\mathbf{x}}+\mathbf{v}_{k}$. The error samples are defined by $e_{k}(i)=\hat{y}_{k}(i)-y_{k}(i)$, and the error vector is defined as $\mathbf{e}_{k}=\mathbf{W}_{k} \hat{\mathbf{x}}-\mathbf{y}_{k}$.

Various image priors may be used. The simplest form involves an exponential function that is quadratic in the voxel values of $\mathbf{x}$, i.e. $\operatorname{Pr}(\hat{\mathbf{x}})=\exp \left(-\hat{\mathbf{x}}^{\mathrm{T}} \mathbf{Q} \hat{\mathbf{x}}\right)$; where $\mathbf{Q}$ is a symmetric, positive definite matrix. Here we use $\mathbf{Q}=\mathbf{C}^{\mathrm{T}} \mathbf{C}$ where $\mathbf{C}$ is the gradient magnitude image operation. Assuming independent slice acquisitions the log-likelihood of the conditional PDF in Equation (3) is the sum of the $l_{2}$-norm of the error vectors over all the slices. Consequently the maximization of the log likelihood function results in the following minimization problem:

$$
\hat{\mathbf{x}}_{M A P}=\arg \min _{\mathbf{x}} \sum_{k=1}^{n}\left\|\mathbf{W}_{k} \hat{\mathbf{x}}-\mathbf{y}_{k}\right\|_{2}^{2}+\lambda\|\mathbf{C} \hat{\mathbf{x}}\|_{2}^{2}
$$

The augmented matrix $\mathbf{W}$ in the linear inverse problem is very large and the classical solution through pseudo-inverse is prohibitive. Instead we use a steepest descent iterative minimization approach. The iterative solution of Equation (4) based on image operators shown in Equation (1) is written as:

$$
\hat{\mathbf{x}}^{n+1}=\hat{\mathbf{x}}^{n}+\alpha \sum_{k=1}^{n} \mathbf{M}_{k}^{\mathbf{T}} \mathbf{S}_{k}^{\mathbf{T}} \mathbf{B}_{k}^{\mathbf{T}} \mathbf{D}_{k}^{\mathbf{T}}\left(\mathbf{y}_{k}-\mathbf{D}_{k} \mathbf{B}_{k} \mathbf{S}_{k} \mathbf{M}_{k} \hat{\mathbf{x}}^{n}\right)-\lambda \mathbf{C}^{\mathrm{T}} \mathbf{C} \hat{\mathbf{x}}^{n},
$$


where $\alpha$ is the step size in the direction of the gradient. The matrices $\mathbf{D}_{k}$, $\mathbf{B}_{k}, \mathbf{S}_{k}$, and $\mathbf{M}_{k}$ and their transposes are exactly interpreted as corresponding image operators. $\mathbf{D}_{k}$ is defined as a resampling operation. $\mathbf{B}_{k}$ is defined as the convolution with a Gaussian kernel resembling the point spread function (PSF) of the MRI signal acquisition process. $\mathbf{C}$ is implemented as a gradient magnitude image operation. $\lambda$ is a weighting coefficient.

The slice selection profile $\mathbf{S}_{k}$ is defined based on the slice selection process. For an arbitrary slice select direction defined by the normal vector of the slice plane equation, the following geometrical equation is obtained for the voxels of slice $k$ (defined by a vector $\boldsymbol{r}$ ) in the slice selection process: $\left|\boldsymbol{\mu}_{s k} . \boldsymbol{r}-s_{0 k}\right|<\Delta s_{k} / 2$; where $\Delta s_{k}$ is the slice thickness, and $s_{0 k}$ is the distance of the slice from the origin. $\boldsymbol{\mu}_{s k}$ specifies the slice (or slice-selection) orientation and is interpreted as the normal vector of the slice plane equation. The normal vector can be obtained in the physical coordinate system based on the so-called direction cosines rotation matrix. Consequently the $\mathbf{S}_{k}$ operation is implemented as rigid 3D rotation with the rotation matrix directly obtained from direction cosines matrix, and its transpose is the inverse (transpose) of the direction cosines matrix.

The motion matrix $\mathbf{M}_{k}$ is implemented as a 6-DOF 3D rigid transformation (including three rotations and three translations). Motion estimation and superresolution volume reconstruction are considered as separable problems. Therefore in the presence of inter-slice motion, iterations of motion correction and volume reconstruction are performed to find $\mathbf{M}_{k}$ and $\mathbf{x}$, respectively. Motion correction can be performed through slice-to-volume registration [1], [3], or based on slice intersections 4. This is not a subject of interest in this article; in order to focus on the performance analysis of volume reconstruction we assume that there is no motion or the motion is known (accurately corrected) in our experiments.

\section{Results}

\subsection{Quantitative Evaluation}

Quantitative evaluation and comparison of the algorithms was carried out using digital brain phantom (DBP) images obtained from the Brainweb database 7 . Thick slice scans in the axial, coronal and sagittal slice select directions were synthetically generated from the high-resolution DBP images by applying operations based on Equation (1). The in-plane resolution of the synthetic scans was $1 \mathrm{~mm}$ and various slice thicknesses were examined between 2 to $8 \mathrm{~mm}$.

Since a reference high-resolution volume is available for the validation dataset (i.e. the original DBP images), the accuracy of reconstruction can be measured quantitatively. Two measures are used here: Mean Absolute Error (MAE) and Peak Signal to Noise Ratio (PSNR). MAE is defined as the mean absolute differences of the voxel intensity values between the reference volume and the reconstructed volume. PSNR is defined in the logarithmic decibel $(\mathrm{dB})$ scale as 
$20 \log _{10}(\mathrm{MAX} / \sqrt{\mathrm{MSE}})$, where MAX is the maximum possible voxel intensity value (4096 in our experiments) and MSE is the mean square error of the voxel intensity values between the reference volume and the reconstructed volume. Lower MAE and higher PSNR indicate more accurate reconstruction.

Fig. 1 shows the MAE and PSNR values computed as a function of the slice thickness of synthesized thick-slice input scans for different volume reconstruction techniques. Four techniques have been considered: AVE is the simplest one and is based on averaging the input scans resampled to the space of the desired high-resolution volumetric image. SDI is a 3-level BSpline SDI approach based on [1, MLE is a non-regularized MLE obtained from the MAP formulation by setting $\lambda=0$, and MAP is the MAP estimation with $\lambda=0.01$. This value was chosen experimentally as a normalization factor between the reconstruction error image and the gradient magnitude of the estimated volume.
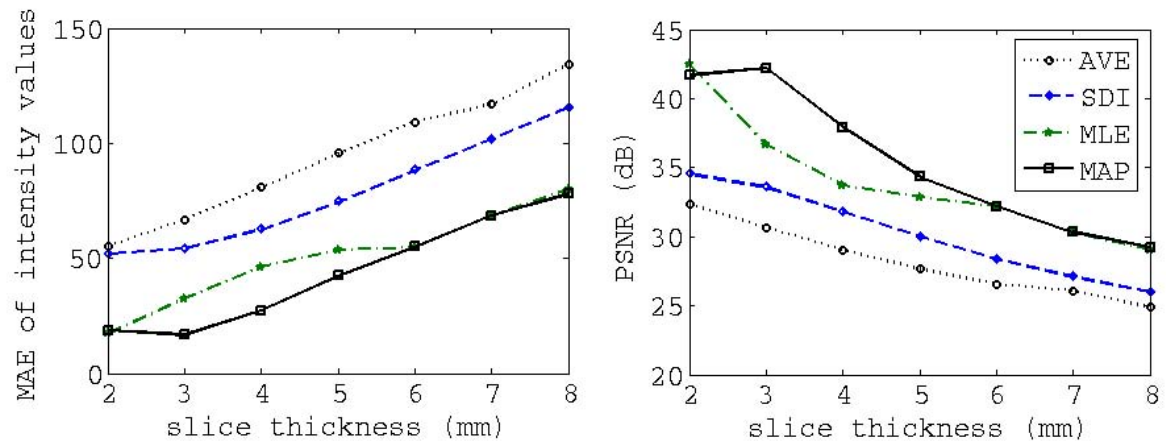

Fig. 1. MAE and PSNR between the ground truth DBP volume and the reconstructed volumes as a function of slice thickness. The measures have been compared for 4 techniques: AVE for averaging the resampled input scans, SDI for BSpline SDI, MLE for non-regularized MLE, and MAP for the developed MAP estimation approach.

The results in Fig. 11indicate that all the techniques perform better than simple averaging. The developed MAP estimation approach outperforms SDI, and is generally more accurate than MLE. The comparison of MAP and MLE at slice thickness $2 \mathrm{~mm}$ indicates that if the slice thickness is not much larger than the in-plane resolution, the prior image model may not provide improvements. On the other hand, when the high-resolution volume space is not densely sampled by the thick-slice scans (i.e. due to large slice thickness and limited number of orthogonal scans), image priors significantly improve the reconstruction accuracy. This is observed for the slice thickness values between 3 to $5 \mathrm{~mm}$. Finally, when the slice thickness is too large (i.e. more than six times larger than the in-plane resolution) image priors in the form of image gradients may not help too much. Improvement of volume estimation is difficult in these cases due to fundamental performance limits in super-resolution reconstruction [8]. 


\subsection{Application to Pediatric and Fetal MRI}

The first application is the estimation of isotropic high-resolution volumes from thick-slice T2-weighted TSE scans of pediatric patients who underwent clinical brain MRI for the evaluation of tuberous sclerosis. As part of the imaging procedure, two T2-weighted TSE volumes (one axial and one coronal) were acquired for each patient using a Siemens Trio 3-Tesla scanner. TSE imaging was performed with $T R=14070 \mathrm{~ms}, T E=89 \mathrm{~ms}$, matrix size of $512 \times 512$, slice thickness of $2 \mathrm{~mm}$, and in-plane resolutions between 0.4 to $0.5 \mathrm{~mm}$. The scanning protocol also involved a high-resolution T1-weighted (T1W) MPRAGE acquisition with $T R=2530 \mathrm{~ms}, T E=3.39 \mathrm{~ms}$, and isotropic resolution of $1 \mathrm{~mm}$.

The acquired TSE scans, as well as the reconstructed volumes and the T1W MPRAGE volume of a 3-year-old child are shown in Fig. 2. The reconstructed volumes in this case have a high isotropic resolution of $0.5 \mathrm{~mm}^{3}$, which is four times better than the slice thickness and two times better than the MPRAGE volume. Visual inspection indicates that the MAP estimated volume is much sharper and has a better contrast as compared to the SDI estimated volume.

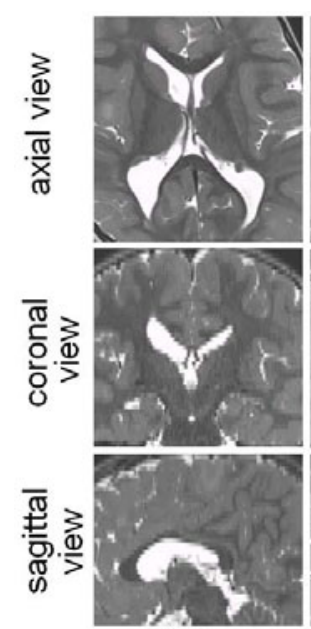

(a)

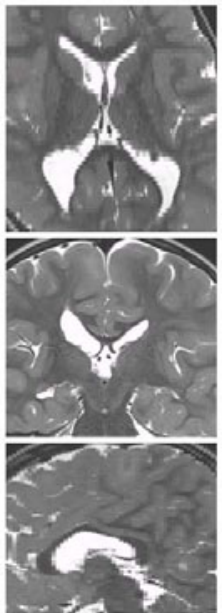

(b)

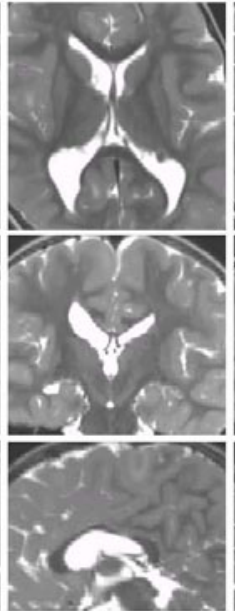

(c)

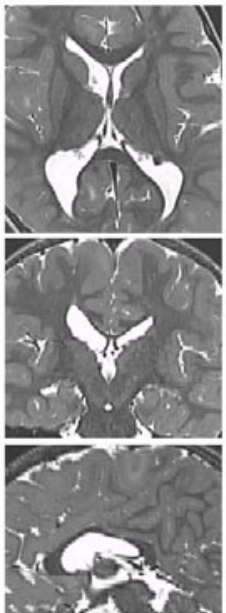

(d)

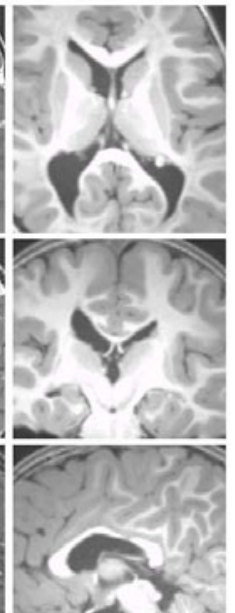

(e)

Fig. 2. Application of the volume reconstruction algorithms to T2-weighted TSE images of a 3-year old child: Two thick-slice TSE scans were acquired and used for reconstruction in the (a) axial and (b) coronal directions; (c) and (d) are the volumes reconstructed using the SDI approach and the MAP estimation approach, respectively, and (e) is the acquired high-resolution T1W MPRAGE volume

We use two sets of measures for comparing the accuracy of volume reconstructions. First we compute the similarity of the reconstructed $\mathrm{T} 2 \mathrm{~W}$ volumes to the acquired high-resolution T1W volume. Normalized mutual information (NMI) is appropriate in this case as it quantifies the nonlinear relationship between 
the intensity values of images with different contrast sources. We also use two sharpness (focus) measures: M1 (the intensity variance measure) and M2 (the energy of image gradient measure). Both measures are monotonic and robust to noise 9]. The variance measure is calculated as the sum of square differences (SSD) between each voxel intensity value and the mean image intensity value. M2 is computed by integrating the magnitude of image gradient at all voxels.

The NMI measure computed as the similarity of T2W volume to the acquired T1W volume was 1.61, 1.71, and 2.00 for the AVE, SDI, and MAP estimation techniques respectively. This indicates that from an information-theoretic viewpoint the intensity values of the MAP estimated volume better match with those of the reference T1W volume. The computed M1 \& M2 sharpness measures were $39092 \& 6.7 \times 10^{11}, 41671 \& 1.0 \times 10^{12}$, and $43139 \& 1.1 \times 10^{12}$ for the AVE, SDI, and MAP estimation techniques respectively. This indicates that the sharpest volumes were obtained from the MAP estimation approach.

The second application of this technique is for fetal MRI. Iterative inter-slice motion correction and volume reconstruction was performed here. Clinical fetal MRI scans were obtained using a 1.5-T TwinSpeed Signa system and an 8-channel phased-array cardiac coil for pregnant patients with diagnosed or suspected cases of fetal anomalies after diagnostic ultrasonography. The input scans involve multiple SSFSE acquisitions in the fetal sagittal, axial and coronal planes with slice thickness between 3 to $5 \mathrm{~mm}$, and in-plane resolution of 0.7 to $0.8 \mathrm{~mm}$.

Fig. 3 shows an example of volumetric fetal brain MRI reconstruction. Note that the reconstructed volume clearly reflects the underlying continuity of tis-

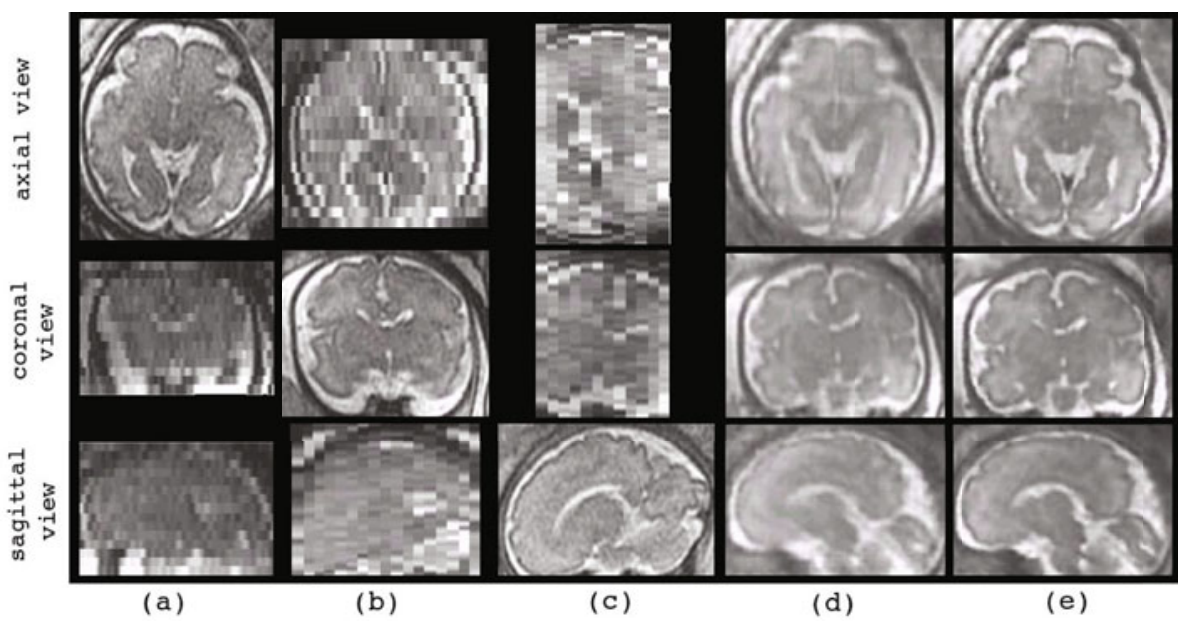

Fig. 3. Application of the volume reconstruction algorithms to a 31.43 week fetus: Three of the six acquired SSFSE scans in fetal axial, coronal, and sagittal planes are shown in (a) to (c) respectively. (d) and (e) show the volumes reconstructed with isotropic resolution of $0.8 \mathrm{~mm}^{3}$ using the SDI approach and the MAP estimation approach, respectively. Note that coherent tissue boundaries present in all three planes of the MAP estimated volume but not in the out-of-plane views of the original scans 
sue structural boundaries in all three planes, whereas the original acquisitions exhibit discontinuous tissue boundaries in the out-of-plane views due to the effect of partial volume averaging. We examined 15 fetal brain MRI datasets and computed sharpness measures. The average improvement in the M1 and M2 sharpness measures with respect to the AVE reconstructed volumes were $8 \%$ and $20 \%$ for the SDI, and $12 \%$ and $42 \%$ for the MAP estimated volumes, respectively.

\section{Conclusion}

We have developed a MAP estimation approach for the reconstruction of isotropic high-resolution volumetric MRI from thick-slice orthogonal scans. This formulation is based on a slice acquisition model, minimizes a cost function of an error norm between the acquired thick-slice scans and the reconstructed volume, and provides a framework for the incorporation of image priors. The results indicate that the MAP estimation approach outperforms the scattered data interpolation techniques, and image priors result in improved accuracy when the slice thickness is 3 to 5 times larger than the in-plane resolution. In addition to fetal and pediatric MRI, this approach can be used in many other MRI applications.

Acknowledgments. This research was supported in part by NIH grants R01 RR021885, R01 GM074068, R01 EB008015, and R43 MH086984.

\section{References}

1. Jiang, S., Xue, H., Glover, A., Rutherford, M., Rueckert, D., Hajnal, J.: MRI of moving subjects using multislice snapshot images with volume reconstruction (SVR): application to fetal, neonatal, and adult brain studies. IEEE Transactions on Medical Imaging 26(7), 967-980 (2007)

2. Greenspan, H., Oz, G., Kiryati, N., Peled, S.: MRI inter-slice reconstruction using super-resolution. Magnetic Resonance Imaging 20(5), 437-446 (2002)

3. Rousseau, F., Glenn, O., Iordanova, B., Rodriguez-Carranza, C., Vigneron, D., Barkovich, J., Studholme, C.: Registration-based approach for reconstruction of high-resolution in utero fetal MR brain images. Acad. Radiol. 13, 1072-1081 (2006)

4. Kim, K., Habas, P., Rousseau, F., Glenn, O., Barkovich, A., Studholme, C.: Intersection based motion correction of multi-slice MRI for $3 \mathrm{~d}$ in utero fetal brain image formation. IEEE Transactions on Medical Imaging 29(1), 146-158 (2010)

5. Park, S.C., Park, M.K., Kang, M.G.: Super-resolution image reconstruction: a technical overview. IEEE Signal Processing Magazine 20(3), 21-36 (2003)

6. Farsiu, S., Robinson, M., Elad, M., Milanfar, P.: Fast and robust multiframe super resolution. IEEE Transactions on Image Processing 13(10), 1327-1344 (2004)

7. Collins, D., Zijdenbos, A., Kollokian, V., Sled, J., Kabani, N., Holmes, C., Evans, A.: Design and construction of a realistic digital brain phantom. IEEE Transactions on Medical Imaging 17(3), 463-468 (1998)

8. Robinson, D., Milanfar, P.: Statistical performance analysis of super-resolution. IEEE Transactions on Image Processing 15(6), 1413-1428 (2006)

9. Subbarao, M., Choi, T., Nikzad, A.: Focusing techniques. Journal of Optical Engineering 32, 2824-2836 (1992) 